\title{
A CERTAIN CLASS OF APPROXIMATION OPERATORS
}

\author{
EMIL C. POPA
}

Abstract. In this paper we are studying the sequences of linear positive operators $\left(P_{n}^{\alpha, \beta}\right)$ defined by means of the umbral calculus. We are proving that the sequence $\left(P_{n}^{\alpha, \beta}\right)$ converges uniformly to the identity operator and we give some results for the rate of convergence for $P_{n}^{\alpha, \beta} f$.

Mathematics subject classification (2000): 41A36, 05A40.

Key words and phrases: Delta operator, basic set, Sheffer polynomials, Cheney-Sharma operator, approximation operators.

\section{REFERENCES}

[1] AgRATINI O., On the rate of convergence of a positive approximation process, Nihonkai Math. J., 11, (2000), 47-56.

[2] Cheney E. W., Sharma A., On a generalization of Bernstein polynomials, Riv.Mat.Univ.Parma 2, 5 (1964), 77-84.

[3] CRĂCIUN M., Approximation operators constructed by means of Sheffer sequences, Rev. d'analyse num. et théorie de l'approx., Tome 30, no. 2, 2001, 135-150.

[4] LuPAŞ A., On the approximation operators of binomial type, Proc. of International Meeting Approximation Theory, Dortmund, 1998, New Developments in Approximation Theory, Birkhäuser-Verlag-Basel, International Series of Numerical Mathematics, I.S.N.M. vol.132, 1999, 175-198.

[5] Mitrinović D. S., Analytic Inequalities, Springer Verlag, Berlin-Heidelberg-New York, 1979.

[6] POPA C. E., Sheffer polynomials and approximations operators, Tamkang J. Math., Tamsui, Taiwan, 34, 2 June (2003), 117-127

[7] Roman S., The umbral calculus, Academic Press, Inc. 1984.

[8] Roman S., Rota G. C., The umbral calculus, Advances in Mathematics, 27, 2 (1978), 95-188.

[9] Rota G. C., KAHNER D. AND OdLYZKo A., On the foundations of combinatorial theory VIII : Finite operator calculus, J.Math. Anal. Appl. 42 (1973), 684-760.

[10] Sablonniere P., Positive Berstein-Sheffer operators, J.Approx. Theory, 83 (1995), 330-341.

[11] StAncu D. D., OCCORSIO M. R., On approximation by binomial operators of Tiberiu Popoviciu type, Revue d'Anlyse Num. et de Théorie de l'Approx., Tome 27 no.1, 1998, 167-181. 